$11-1-2013$

\title{
Comparison of Parameters of Lognormal Distribution Based On the Classical and Posterior Estimates
}

Raja Sultan

University of Kashmir, Srinagar, India, hamzasultan18@yahoo.com

S. P.Ahmad

University of Kashmir, Srinagar, India, sprvz@yahoo.com

Follow this and additional works at: http://digitalcommons.wayne.edu/jmasm

Part of the Applied Statistics Commons, Social and Behavioral Sciences Commons, and the Statistical Theory Commons

\section{Recommended Citation}

Sultan, Raja and Ahmad, S. P. (2013) "Comparison of Parameters of Lognormal Distribution Based On the Classical and Posterior Estimates," Journal of Modern Applied Statistical Methods: Vol. 12 : Iss. 2 , Article 18.

DOI: $10.22237 /$ jmasm/1383279420

Available at: http://digitalcommons.wayne.edu/jmasm/vol12/iss2/18

This Regular Article is brought to you for free and open access by the Open Access Journals at DigitalCommons@WayneState. It has been accepted for inclusion in Journal of Modern Applied Statistical Methods by an authorized editor of DigitalCommons@WayneState. 


\section{Comparison of Parameters of Lognormal Distribution Based On the Classical \& Posterior Estimates}

\author{
Raja Sultan \\ University of Kashmir \\ Srinagar, India
}

\author{
S. P. Ahmad \\ University of Kashmir \\ Srinagar, India
}

Lognormal distribution is widely used in scientific field, such as agricultural, entomological, biology etc. If a variable can be thought as the multiplicative product of some positive independent random variables, then it could be modelled as lognormal. In this study, maximum likelihood estimates and posterior estimates of the parameters of lognormal distribution are obtained and using these estimates we calculate the point estimates of mean and variance for making comparisons.

Keywords: Lognormal distribution, maximum likelihood estimation, posterior estimates \& R software

\section{Introduction}

Aitchison \& Brown (1957) have given a very comprehensive treatment of lognormal distribution. The lognormal distribution arises in various different contexts such as in physics (distribution of particles due to pulverisation); economics (income distributions); biology (growth of organisms), etc. Epstein (1947), Brownlee (1949), Delaporte (1950), Moroney (1951) describes various applications of lognormal distribution to physical and industrial processes, textile research and quality control. In the context of life testing and reliability problems, the lognormal distribution answers a criticism sometimes raised against the use of normal distribution (ranging from $-\infty$ to $+\infty$ ) as a model for the failure time distribution which must range from 0 to $\infty$.

A random variable $\mathrm{X}$ is said to have a lognormal distribution if $U=\log _{e} X$ has normal distribution with mean $\mu$ and variance $\sigma^{2}$. Thus, the pdf of lognormal distribution is given by

Dr. Sultan is a research scholar in the Department of Statistics. Email him at: hamzasultan18@yahoo.com.Dr. Ahmad is an Assistant Professor of in the Department of Statistics.Email him at: sprvz@yahoo.com. 


\section{SULTAN \& AHMAD}

$$
f(x)=\frac{1}{\sqrt{2 \pi} \sigma x} \exp \left(-\frac{1}{2 \sigma^{2}}(\log x-\mu)^{2}\right) \quad,-\infty<\mu<\infty, \sigma^{2}>0, \quad 0 \leq x<\infty
$$

The likelihood function of the random sample $\left(\mathrm{x}_{1}, \mathrm{x}_{2}, \mathrm{x}_{3}, \ldots, \mathrm{x}_{\mathrm{n}}\right)^{\mathrm{T}}$ would be

$$
L\left(\mu, \sigma^{2} \mid x\right)=\left(\frac{1}{\sqrt{2 \pi} \sigma x}\right)^{n} \exp \left(-\frac{1}{2 \sigma^{2}} \sum_{i=1}^{n}\left(\log x_{i}-\mu\right)^{2}\right)
$$

The mean and variance of the lognormal distribution are given by

$$
E(X)=\alpha_{1}=\exp \left(\mu+\frac{\sigma^{2}}{2}\right)
$$

and

$$
V(X)=\beta_{1}=\exp \left(2 \mu+\sigma^{2}\right)\left(\exp \left(\sigma^{2}\right)-1\right)
$$

\section{Maximum Likelihood Estimators}

Maximum Likelihood is a popular estimation technique for many distributions because it picks the values of the distribution's parameters that make the data "more likely" than any other values of the parameters would make them. This is accomplished by maximizing the likelihood function of the parameters given the data.

Consider the estimation of the parameters $\alpha 1$ and $\beta 1$. Let $U_{i}=\log x_{i}, i=1,2, \ldots, n$. Then using the fact that $\left(\mathrm{U}_{1}, \mathrm{U}_{2}, \ldots, \mathrm{U}_{\mathrm{n}}\right)$ is a random

sample from Normal distribution with parameters $\left(\mu, \sigma^{2}\right)$. The mle of $\mu$ and $\sigma^{2}$ first are given by

$$
\hat{\mu}=\frac{1}{n} \sum_{i=1}^{n} U_{i}=\bar{U}
$$

and 


$$
\hat{\sigma}^{2}=\frac{1}{n} \sum_{i=1}^{n}\left(U_{i}-\bar{U}\right)^{2}
$$

The mle of $\alpha_{1}$ and $\beta_{1}$ are given by

$$
\hat{\alpha}_{1}=\exp \left(\hat{\mu}+\frac{\hat{\sigma}^{2}}{2}\right)
$$

and

$$
\hat{\beta}_{1}=\exp \left(2 \hat{\mu}+\hat{\sigma}^{2}\right)\left(\exp \left(\hat{\sigma}^{2}\right)-1\right)
$$

\section{Posterior estimation of the parameter}

Again, consider the estimation of the parameters $\alpha_{1}$ and $\beta_{1}$. First obtain the posterior estimates of $\mu$ and $\sigma^{2}$ and then simultaneously the posterior estimates for $\alpha_{1}$ and $\beta_{1}$ will be obtained. Laplace (1774) found that it worked exceptionally well to simply always choose the prior probability distribution for the parameter(s) of the model to be constant on the parameter space.

The joint prior pdf for $\mu$ and $\sigma^{2}$ considered is

$$
P\left(\mu, \sigma^{2}\right) \propto 1
$$

According to Bayes theorem, Joint posterior density of $\mu$ and $\sigma^{2}$ is given by

$$
\begin{aligned}
& \text { Posterior density } \propto \text { prior density* likelihood } \\
& \pi\left(\mu, \sigma_{-}^{2} \underset{-}{x}\right) \propto P\left(\mu, \sigma^{2}\right) \cdot P\left(\mu,\left.\sigma_{-}^{2}\right|_{-} ^{x}\right)
\end{aligned}
$$

From equation (2) and (9) the joint posterior density of $\mu$ and $\sigma^{2}$ is given by

$$
\pi\left(\mu, \sigma^{2} \mid \underset{-}{x}\right) \propto\left[\frac{1}{\sqrt{2 \pi \sigma x}}\right]^{n} \exp \left\{\frac{-1}{2 \sigma^{2}} \sum_{i=1}^{n}\left(\log x_{i}-\mu\right)^{2}\right\}
$$




$$
\pi\left(\mu, \sigma^{2} \mid \underset{-}{x}\right)=\frac{c}{\left(\sigma^{2}\right)} \exp \left(\frac{-\beta}{2 \sigma^{2}}\right) \exp \left\{\frac{-n}{2 \sigma^{2}}\left(\mu-\frac{\sum_{i=1}^{n} \log x_{i}}{n}\right)^{2}\right\}
$$

where $\beta=\sum_{i=1}^{n}\left(\log x_{i}\right)^{2}-\frac{\left(\sum_{i=1}^{n} \log x_{i}\right)^{2}}{n}$ and c is a normalizing constant. Lindley (1961) explained if $P(\theta)$ be the prior and $P\left({ }_{-} \mid \theta\right)$ be the likelihood, the posterior pdf $P\left(\theta \mid{ }_{-}^{x}\right)$ is given by $P(\theta \mid \underset{-}{x})=c P(\theta) . P\left({ }_{-}^{x} \mid \theta\right)$, where $\mathrm{c}$ is the normalizing constant. Then the value of $\mathrm{c}$ is obtained by $c=\left[\int P(\theta) \cdot P(\underset{-}{x} \mid \theta) d \theta\right]^{-1}$

Therefore, c can be obtained by

$$
\begin{aligned}
& c^{-1}=\int_{0}^{\infty} \int_{-\infty}^{\infty} \pi\left(\mu, \sigma^{2} \mid \underset{-}{x}\right) d \mu d \sigma^{2} \\
& c^{-1}=\int_{0}^{\infty} \int_{-\infty}^{\infty} \frac{1}{\left(\sigma^{2}\right)^{n / 2}} \exp \left(\frac{-\beta}{2 \sigma^{2}}\right) \exp \left\{\frac{-n}{2 \sigma^{2}}\left(\mu-\frac{\sum_{i=1}^{n} \log x_{i}}{n}\right)\right\} d \mu d \sigma^{2} \\
& \text { Using the transformation } t=\frac{\sqrt{n}\left(\mu-\frac{\sum_{i=1}^{n} \log x_{i}}{n}\right)}{\sigma}
\end{aligned}
$$




\section{COMPARISON OF PARAMETERS OF LOGNORMAL DISTRIBUTION}

$$
\begin{gathered}
c^{-1}=\sqrt{\frac{2 \pi}{n} \int_{o}^{\infty} \frac{\exp \left(\frac{-\beta}{2 \sigma^{2}}\right)}{\left(\sigma^{2}\right)^{n-1 / 2}} d \sigma^{2}} \\
c^{-1}=\sqrt{\frac{2 \pi}{n}} \frac{\Gamma\left(\frac{n-3}{2}\right)}{\left(\frac{\beta}{2}\right)^{\frac{n-3}{2}}} \\
\Rightarrow c=\sqrt{\frac{n}{2 \pi}} \frac{(\beta)^{\frac{n-3}{2}}}{2^{\frac{n-3}{2}} \Gamma\left(\frac{n-3}{2}\right)}
\end{gathered}
$$

From the equation (10)

$$
\pi\left(\mu, \sigma^{2} \mid \underset{-}{x}\right)=\sqrt{\frac{n}{2 \pi}} \frac{(\beta)^{\frac{n-3}{2}}}{2^{\frac{n-3}{2}} \Gamma\left(\frac{n-3}{2}\right)\left(\sigma^{2}\right)^{n / 2}} \exp \left(\frac{-\beta}{2 \sigma^{2}}\right) \exp \left\{\frac{-n}{2 \sigma^{2}}\left(\mu-\frac{\sum_{i=1}^{n} \log x_{i}}{n}\right)^{2}\right\}
$$

\section{Marginal posterior densities of $\mu$ and $\sigma^{2}$}

The marginal density of $\mu$ is obtained by integrating out $\sigma^{2}$ from (12) and is given as

$$
\pi(\mu \underset{-}{x})=\int_{0}^{\infty} \pi\left(\mu, \sigma_{-}^{2} \underset{-}{x}\right) d \sigma^{2}
$$




\section{SULTAN \& AHMAD}

$$
\begin{aligned}
& \pi(\mu \mid \underset{-}{x})=c \int_{0}^{\infty} \frac{1}{\left(\sigma^{2}\right)^{n / 2}} \exp \left[-\frac{1}{2 \sigma^{2}}\left\{\beta+n\left(\mu-\frac{\sum_{i=1}^{n} \log x_{i}}{n}\right)^{2}\right] d \sigma^{2}\right. \\
& \pi(\mu \mid \underset{-}{x})=c \frac{\Gamma\left(\frac{n}{2}-1\right) 2^{2^{\frac{n}{2}-1}}}{\left[\beta+n\left(\mu-\frac{\sum_{i=1}^{n} \log x_{i}}{n}\right)^{2}\right]^{\frac{n}{2}-1}} \\
& \pi(\mu \mid \underset{-}{x})=\sqrt{\frac{n}{\beta}} \frac{1}{B\left(\frac{1}{2}, \frac{n-3}{2}\right)\left[1+\frac{n}{\beta}\left(\mu-\frac{\sum_{i=1}^{n} \log x_{i}}{n}\right)^{2}\right]^{\frac{n-2}{2}}}
\end{aligned}
$$

The marginal density of $\sigma^{2}$ is obtained by integrating the joint posterior density of $\mu$ and $\sigma^{2}$ given in (12) over the range of $\mu$. It is given as

$$
\begin{gathered}
\pi\left(\sigma^{2} \mid \underset{-}{x}\right)=c \int_{-\infty}^{\infty} \frac{1}{\left(\sigma^{2}\right)^{n / 2}} \exp \left(\frac{-\beta}{2 \sigma^{2}}\right) \exp \left\{-\frac{n}{2 \sigma^{2}}\left(\mu-\frac{\sum_{i=1}^{n} \log x_{i}}{n}\right)^{2}\right\} d \mu \\
\pi\left(\sigma^{2} \mid \underset{-}{x}\right)=\frac{c \exp \left(\frac{-\beta}{2 \sigma^{2}}\right)^{\infty}}{\left(\sigma^{2}\right)^{n / 2}} \exp \left\{-\frac{n}{2 \sigma^{2}}\left(\mu-\frac{\sum_{i=1}^{n} \log x_{i}}{n}\right)^{2}\right\} d \mu
\end{gathered}
$$




\section{COMPARISON OF PARAMETERS OF LOGNORMAL DISTRIBUTION}

$$
\begin{gathered}
\pi\left(\sigma^{2} \mid x\right)=c \frac{\exp \left(\frac{-\beta}{2 \sigma^{2}}\right) \sqrt{2 \pi}}{\left(\sigma^{2}\right)^{n / 2} \sqrt{n}} \sqrt{\sqrt{n}} \\
\pi\left(\sigma_{-}^{2} \mid x\right)=\frac{\exp \left(\frac{-\beta}{2 \sigma^{2}}\right) \beta^{\frac{n-3}{2}}}{\left(\sigma^{2}\right)^{n / 2} 2^{\frac{n-3}{2}} \Gamma\left(\frac{n-3}{2}\right)}
\end{gathered}
$$

\section{Posterior estimates of $\mu$ and $\sigma^{2}$}

The marginal density of $\mu$ is given in (13) is a student's t pdf. Thus the posterior estimates of $\mu$ is given as

$$
\mu^{*}=E(\mu \mid \underset{-}{x})=\sqrt{\frac{n}{\beta}} \frac{1}{B\left(\frac{1}{2}, \frac{n-3}{2}\right)} \int_{-\infty}^{\infty} \frac{\mu d \mu}{\left.1+\frac{n}{\beta}\left(\mu-\frac{\sum \log x_{i}}{n}\right)^{2}\right]^{\frac{n-2}{2}}}
$$

Using the transformation $t=\sqrt{\frac{n}{\beta}}\left(\mu-\frac{\sum_{i=1}^{n} \log x_{i}}{n}\right) \sqrt{n-3}$

$$
\mu^{*}=\frac{\sum_{i=1}^{n} \log x_{i}}{n \sqrt{n-3} B\left(\frac{1}{2}, \frac{n-3}{2}\right)} \int_{-\infty}^{\infty} \frac{d t}{\left[1+\frac{t^{2}}{n-3}\right]^{\frac{n-2}{2}}}
$$




\section{SULTAN \& AHMAD}

$$
\mu^{*}=\frac{\sum_{i=1}^{n} \log x_{i}}{n}
$$

which is the posterior estimate for $\mu$ under uniform prior. Now the posterior estimate of $\sigma^{2}$ can be obtained from equation (14) as

$$
\begin{gathered}
\sigma^{* 2}=\int_{0}^{\infty} \frac{\sigma^{2} \exp \left(\frac{-\beta}{2 \sigma^{2}}\right) \beta^{\frac{n-3}{2}}}{\left(\sigma^{2}\right)^{\frac{n-1}{2}} 2^{\frac{n-3}{2}} \Gamma\left(\frac{n-3}{2}\right)} d \sigma^{2} \\
\sigma^{* 2}=\frac{\beta^{\frac{n-3}{2}}}{2^{\frac{n-3}{2}} \Gamma\left(\frac{n-3}{2}\right)^{\infty}} \frac{\exp \left(\frac{-\beta}{2 \sigma^{2}}\right)}{\left(\sigma^{2}\right)^{\frac{n-3}{2}}} d \sigma^{2} \\
\sigma^{* 2}=\frac{\beta}{n-5}
\end{gathered}
$$

Thus, the posterior estimates of $\alpha_{1}$ and $\beta_{1}$ are given by

$$
\alpha_{1}^{*}=\exp \left[\mu^{*}+\frac{\sigma^{* 2}}{2}\right]=\exp \left[\frac{\sum_{i=1}^{n} \log x_{i}}{n}+\frac{\beta}{2(n-5)}\right]
$$

and

$$
\beta_{1}^{*}=\exp \left[2 \mu^{*}+\sigma^{* 2}\right]\left[\exp \left(\sigma^{* 2}\right)-1\right]
$$




$$
\beta_{1}^{*}=\exp \left[2 \frac{\sum_{i=1}^{n} \log x_{i}}{n}+\frac{\beta}{n-5}\right]\left[\exp \left(\frac{\beta}{n-5}\right)-1\right]
$$

\section{Simulation study and discussion}

The estimates of the mean and variance using MLE and Bayesian estimation was obtained above. Next to obtain is the numerical relationship of point estimates using true value of the parameters, MLE and Bayesian estimation.

In this study, samples of 10, 20, 30, 40 and 50 observations were generated from lognormal pdf with parameters $\mu=2$ and $\sigma=1$. The simulations were done in R Software. The mean and variance were calculated to compare the methods of estimation. The results are presented in Table 1.

In Table 1, when point estimates of lognormal distribution are compared using true values of parameters with MLE and Bayesian estimation (by using uniform prior), the best estimator is the Maximum Likelihood (MLE) because it has the minimum variance.

Table 1. Point estimates of lognormal distribution compared using true values of parameters with MLE and Bayesian estimation

\begin{tabular}{ccccccc}
\hline $\mathbf{n}$ & \multicolumn{2}{c}{ True values } & \multicolumn{2}{c}{ MLE } & \multicolumn{2}{c}{ Posterior estimates } \\
\hline & Mean & Variance & Mean & Variance & Mean & Variance \\
& $\left(\alpha_{1}\right)$ & $\left(\beta_{1}\right)$ & $\left(\hat{\alpha}_{1}\right)$ & $\left(\hat{\beta}_{1}\right)$ & $\left(\alpha_{1}^{*}\right)$ & $\left(\beta_{1}^{*}\right)$ \\
\hline 10 & & & 9.9004 & 21.336 & 9.1225 & 62.1358 \\
20 & & & 12.6952 & 72.127 & 9.8447 & 130.857 \\
30 & \multirow{2}{*}{12.1825} & 255.011 & 12.6655 & 52.1804 & 10.5913 & 70.6613 \\
40 & & & 12.6452 & 56.9317 & 10.2974 & 71.6757 \\
50 & & & 20.4039 & 267.461 & 12.5356 & 356.339 \\
\hline
\end{tabular}

\section{References}

Aitchison, J., \& Brown, J. A. C. (1957). The lognormal distribution: With special reference to its uses in economics. Cambridge: University Press. 


\section{SULTAN \& AHMAD}

Brownlee, K. A. (1949). Industrial experimentation. 2nd ed. Brooklyn: Chemical Pub. Co.

Delaporte, P. (1950). Etude statistique sur les proprietes des fontes. Revue De L'institut International De Statistique / Review Of The International Statistical Institute, (3/4), 161. doi:10.2307/1401035

Epstein. B. (1947). The mathematical description of certain breakage mechanism leading to the logarithmic-normal distribution. Journal of Franklin Institute, 244, 471-477.

Laplace, P. S. (1774). Mémorie sur la probabilitié des causes par les événemens. Académie royale des sciences (France), 6, 621-656.

Lindley, D. V. (1961). Introduction to probability and statistics from a Bayesian viewpoint: Part 2, Inference, Aberystwyth: University College of Wales. Moroney, M. J. (1951). Facts from figures. Baltimore, MD: Penguin Books. 